\title{
Resonance enhancement of x-rays and fluorescence yield from marker layers in thin films
}

\author{
S. K. Ghose ${ }^{\dagger}$ and B. N. Dev* \\ Institute of Physics, Sachivalaya Marg, Bhubaneswar - 751 005, India \\ Ajay Gupta \\ Inter University Consortium of DAEF, University Campus, Khandwa Road, Indore - 452001, India
}

(Received 11 April 2001; published 20 November 2001)

\begin{abstract}
Resonance enhancement of $\mathrm{x}$ rays in a thin film and fluorescence emission from embedded marker layers within the film have been studied. With embedded marker layers of Ti, Fe, and W at different depths in a thin Si film on a Au-coated Si substrate, it has been shown that the position of a marker layer throughout the depth of the film can be unambiguously determined with a precision better than $0.5 \mathrm{~nm}$. In this example, fieldintensity enhancement upto 16 times have been observed. Field enhancement gives rise to enhanced sensitivity. The usefulness of this resonance-enhanced x-ray fluorescence spectrometry in the study of diffusion with marker layers in thin films including polymers and nanocomposites has been elucidated.
\end{abstract}

DOI: 10.1103/PhysRevB.64.233403

Physical phenomena such as, total external reflection, ${ }^{1}$ interference fringes in reflection from layers on a substrate, ${ }^{2}$ formation of evanescent, ${ }^{3,4}$ and standing waves, ${ }^{5}$ and resonance enhancement of $x$ rays in thin films on substrates ${ }^{6-8}$ are observed when $\mathrm{x}$ rays are incident at grazing angles on flat surfaces of materials. Odd-order resonance enhancements of $\mathrm{x}$ rays have been observed by Wang et al. in a Langmuir-Blodgett (LB) layer ${ }^{6}$ and Dev et al. observed both odd and even order enhancements in a polymer blend layer. ${ }^{7}$ The depth distribution of the two polymer components in the blend was determined by measuring the $\mathrm{x}$-ray fluorescence yield from specific (marker) atoms in one polymer component as a function of angle of incidence.

Marker layers are usually used in the study of polymerpolymer interdiffusion in thin films. For example, diffusion coefficients have been determined by placing a marker layer of Au between two polystyrene layers of different molecular weights and measuring the shifts of the marker layer position due to polymer interdiffusion by Rutherford backscattering spectrometry (RBS). ${ }^{9}$ X-ray reflectivity (XRR) is also used to study diffusion of polymers of different molecular weights with the help of heavy atom marker layers. ${ }^{10}$ While the RBS technique has poor depth resolution, the disadvantage with XRR is that the heavy atom layer has to be thick enough to remain as uniform layer to give a good electron density contrast in the polymer. Resonance-enhanced x-ray fluorescence spectrometry (REXFS), as shown here, would be a better choice for diffusion studies with marker layers. In these experiments fluorescence from marker atoms are detected. The pattern of fluorescence yield variation as a function of angle of incidence contains the information about the marker depth. In the experiment by Wang et al., fluorescence signal was collected from a loosely packed biatomic layer, while the number of fluorescing atoms involved in the experiment by Dev et al. was only $\sim 1 \times 10^{15}$ atoms $/ \mathrm{cm}^{2}$. REXFS offers high sensitivity $\left(\sim 10^{13}\right.$ atoms $/ \mathrm{cm}^{2}$ or better) and high depth resolution $(\sim 0.2 \mathrm{~nm})$.

For resonance enhancement of $\mathrm{x}$ rays, various plots of intensities in earlier works ${ }^{6-8}$ as well as in the inset of Fig. 1(b) show that the X-ray field intensities at depths equidistant from the middle of the film are equal. It may appear from the
PACS number(s): 61.10.Eq, 68.60.-p, 07.85.-m

plots that a marker layer placed at either of these two equidistant positions would give rise to the same fluorescence yield, and consequently by fluorescence yield measurements their positions could not be distinguished. However, this is not true. The field intensities are equal at these positions only at specific angles. Here we demonstrate that fluorescence yields from marker atoms placed at depths equidistant from the middle of the film, detected over an angular range, have quite different profiles and there is no ambiguity in the detection of these positions. We demonstrate this by measuring fluorescence yield from thin $(\sim 1 \mathrm{~nm})$ embedded marker layers of $\mathrm{Ti}, \mathrm{Fe}$, and $\mathrm{W}$ at different depths within a thin Si film $(\sim 50 \mathrm{~nm})$ on a Au-coated Si substrate. Ti, Fe, and W layers were embedded at approximate depths of 12.5, 25.0, and $37.5 \mathrm{~nm}$, respectively. That is, the $\mathrm{Ti}$ and the $\mathrm{W}$ layers are equidistant from the position of the Fe layer. Results clearly show that any marker position in the film can be determined unambiguously. Thus the movement of a marker layer throughout the layer thickness can be precisely determined as needed in a diffusion experiment.

We first present the computation of the theoretical field intensities from a model system-a Si thin film on a Aucoated Si substrate. We compute the angular dependence of the field intensities at depths where we plan to embed the marker layers. The detailed theoretical description is followed from the work of Dev et al. ${ }^{7}$

If we consider a multilayer as schematically shown in the inset of Fig. 1(a), where $E_{0}^{t}$ and $E_{0}^{r}$ represent the incident and the reflected $E$ fields outside the top layer, respectively and $E_{j}^{t}(0)$ and $E_{j}^{r}(0)$ represent the transmitted and the reflected $E$ fields at the top of the $j$ th layer. Then the total $E$ field at the position $\mathbf{r}$ within the $j$ th layer is given by

$$
\mathbf{E}_{j}^{T}(\mathbf{r})=\mathbf{E}_{j}^{t}(\mathbf{r})+\mathbf{E}_{j}^{r}(\mathbf{r})
$$

and the field intensity (for $s$ polarization) at a given depth $z$ from the top of the $j$ th layer is

$$
I_{j}(\theta, z)=\left|E_{j}^{T}(\mathbf{r})\right|^{2} .
$$

Reflectivity outside the surface is given by $R=\left|E_{0}^{r} / E_{0}^{t}\right|^{2}$. $E_{j}^{t}$ 's and $E_{j}^{r}$ 's and in turn $R$ and $I_{j}$ 's are computed using a 


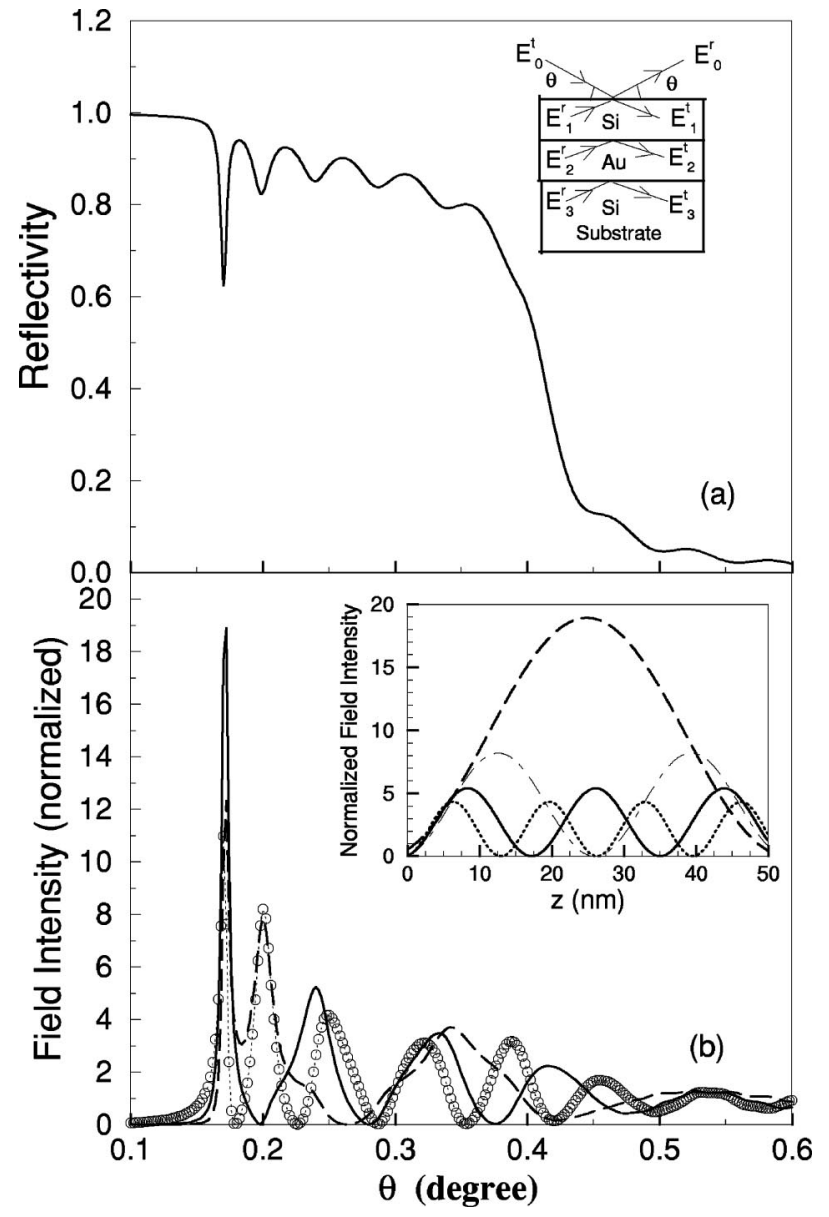

FIG. 1. Theoretical reflectivity $[\mathrm{R}(\theta)]$ and field intensity $\left[I_{1}(\theta, z)\right]$ curves for a $\mathrm{Si}(50 \mathrm{~nm}) / \mathrm{Au}(70 \mathrm{~nm}) / \mathrm{Si}$ (substrate) system. The inset in (a) schematically shows the $\mathrm{Si} / \mathrm{Au} / \mathrm{Si}$ system with reflected and transmitted $E$ fields. (a) Reflectivity, (b) the angular dependence of the field intensities at three depth positions within the top silicon layer, $\frac{1}{4}$ th $[I(\theta, z=12.5 \mathrm{~nm})](\bigcirc . . \bigcirc . . \bigcirc)$, middle $[I(\theta, z=25.0 \mathrm{~nm})](-)$, and $\frac{3}{4}$ th $[I(\theta, z=37.5 \mathrm{~nm})](---)$. Inset in (b) shows the field intensities $I(z)$ within the top Si layer for the angles of incidence $(\theta)$ corresponding to the first, second, third and fourth minima in reflectivity, i.e, at $\theta_{1}=0.173^{\circ}$ $(---), \quad \theta_{2}=0.2^{\circ}(----), \quad \theta_{3}=0.241^{\circ}(-)$, and $\theta_{4}=0.289^{\circ}$ $(\ldots \ldots \ldots)$, respectively. First through fourth order resonance enhancements take place at these angles.

recursion relation ${ }^{7,8,11}$ with the knowledge of the layer thickness $d_{j}$ 's, interface roughness $\sigma_{j}$ 's and the dielectric functions $\epsilon_{j}$ 's, where $\epsilon_{j}$ is given by

$$
\epsilon_{j}=1-2 \delta_{j}-i 2 \beta_{j}
$$

In Eq. (3) $\delta_{j}$ and $\beta_{j}$ are related to the electron density and the linear absorption coefficient of the $j$ th layer, respectively.

The transmitted field intensity, $\left|E_{1}^{t}\right|^{2}$, is an oscillatory function of $\theta$ and may be quite large in comparison to the incident intensity, $\left|E_{0}^{t}\right|^{2}$, under appropriate conditions giving rise to large enhancements ${ }^{7}$ in $I_{j}(\theta, z)$ at specific $\theta$ values. Resonance enhancement occurs at these specific angles. This usually happens within a layer of low electron density (e.g., $\mathrm{Si}$, polymer, LB layer etc.) when it lies on a layer of higher electron density (e.g., Au).

Let us discuss the results of calculations of various quantities using an example. We take a three layer system: $\mathrm{Si} / \mathrm{Au} /$ $\mathrm{Si}$, schematically shown in the inset of Fig. 1(a). In this computation we have used the following parameters: $E_{\gamma}$ (energy of the incident $\mathrm{x}$ rays $)=11.1 \mathrm{keV}, \epsilon_{1}=1-(3.96$ $\left.\times 10^{-6}\right)-\mathrm{i}\left(4.87 \times 10^{-8}\right)$ (silicon), $\epsilon_{2}=1-\left(2.37 \times 10^{-5}\right)-$ $i\left(1.51 \times 10^{-6}\right)$ (gold), $\epsilon_{3}=\epsilon_{1}, d_{1}=50 \mathrm{~nm}$ and $d_{2}=70 \mathrm{~nm}$, $\sigma_{0}=\sigma_{1}=\sigma_{2}=0$. The critical angles, $\theta_{c}=\sqrt{2 \delta}$, are $\theta_{c}(\mathrm{Si})$ $=0.161^{\circ}$ and $\theta_{c}(\mathrm{Au})=0.394^{\circ}$. For $\theta<\theta_{c}(\mathrm{Si})$, incident $\mathrm{x}$ rays will undergo total external reflection from the silicon layer and an evanescent wave will be present in the layer. For $\theta>\theta_{c}(\mathrm{Si})$ the incident $\mathrm{x}$ rays penetrate into the silicon layer and get reflected strongly by the Au layer up to $\theta=\theta_{c}(\mathrm{Au})$. At $\theta_{c}(\mathrm{Si})<\theta<\theta_{c}(\mathrm{Au})$ a part of the x-ray beam, already reflected from the Au layer, is reflected back into the silicon layer from the silicon/air interface. The constructive interference between this beam and the incident beam causes enhancement of field intensity in the silicon layer.

For this system the reflectivity $R(\theta)$ is shown as a function of grazing angle of incidence $(\theta)$ in Fig. 1(a). Field intensity enhancement within the Si layer occurs at those angles where there are minima in the reflectivity curve. The field intensities $I(z)$ within the top Si layer for the angles of incidence $(\theta)$ corresponding to the first, second, third and fourth minima in reflectivity are shown in the inset of Fig. 1(b). We notice that standing wave patterns are generated within the layer. This also shows that the intensity is enhanced within the layer, the enhancement being the largest for the first order, which is about 19 times. We notice from the inset of Fig. 1(b) that second, third and fourth orders have 2, 3, and 4 antinodes, respectively, formed within the layer, with decreasing intensity. In Fig. 1(b) we present the angular dependence of the field intensity at three positions within the top silicon layer, $\frac{1}{4}$ th, middle, and $\frac{3}{4}$ th. Although the field intensities at the $\frac{1}{4}$ th position and the $\frac{3}{4}$ th position are equal at specific angles $\left(\theta_{1}, \theta_{2}, \theta_{3}, \theta_{4}\right)$ where resonance enhancements occur, as shown in the inset, the overall angular dependences are clearly different. So these equidistant positions from the middle of the film can be easily distinguished from each other.

In our experiment we monitor the field intensity by detecting fluorescence yield from $\mathrm{Ti}, \mathrm{Fe}$, and $\mathrm{W}$ atoms embedded as thin marker layers at $z \approx 12.5,25.0$, and $37.5 \mathrm{~nm}$, respectively, within a $50 \mathrm{~nm}$ thick Si film.

The samples were prepared under ultrahigh vacuum condition (base pressure: $3 \times 10^{-9} \mathrm{mbar}$ ) with an $e$-beam coating unit. Six crucibles were used to deposit all the elements. First a thin film of $\mathrm{Cr}(20 \mathrm{~nm})$ was deposited on the Si substrate and then $70 \mathrm{~nm}$ of $\mathrm{Au}$ was deposited on the $\mathrm{Cr}$ layer. $\mathrm{Cr}$ is coated to increase the sticking of $\mathrm{Au}$ on the substrate. Then $\mathrm{Si}$ and the marker layers of $\mathrm{W}, \mathrm{Fe}$, and $\mathrm{Ti}$ were deposited to make the film structure as follows: $\mathrm{Si}(12.5) / \mathrm{Ti}(1.0) / \mathrm{Si}(12.5) / \mathrm{Fe}(1.0) / \mathrm{Si}(12.5) / \mathrm{W}(1.0) /$ $\mathrm{Si}(12.5) / \mathrm{Au}(70) / \mathrm{Cr}(20) / \mathrm{Si}$ (substrate). The numbers in the parenthesis indicate the nominal thickness in $\mathrm{nm}$. The rate of 
deposition for $\mathrm{Si}, \mathrm{Cr}$, and $\mathrm{Au}$ was $0.02 \mathrm{~nm} / \mathrm{sec}$ and that for the three marker layers ( Ti, Fe, and W) was $0.01 \mathrm{~nm} / \mathrm{sec}$.

$\mathrm{X}$-ray reflectivity and fluorescence measurements were performed using synchrotron $\mathrm{x}$ radiation in HASYLAB at the RÖMO I bending magnet beam line. A monochromatic beam $(\lambda=0.1116 \mathrm{~nm})$ was obtained using a $\mathrm{Si}(333)$ and $\mathrm{Si}(511)$ double crystal monochromator. The slit widths parallel and perpendicular to the sample surface were $3.5 \mathrm{~mm}$ and $60 \mu \mathrm{m}$, respectively. The sample size was 25 $\times 20 \mathrm{~mm}^{2}$. Beam divergence was $0.005^{\circ}$. The scattering geometry was that for $s$ polarization. A $\mathrm{NaI}(\mathrm{Tl})$ detector detected the specularly reflected beam and a $\mathrm{Si}(\mathrm{Li})$ detector detected fluorescence from $\mathrm{Ti}, \mathrm{Fe}$, and $\mathrm{W}$ layers, excited by the $E$ field in the sample. The average exit angle $\alpha$ [the inclination of the $\mathrm{Si}(\mathrm{Li})$ detector with respect to the sample surface] for fluorescent photons was $25^{\circ}$. Measurement was made up to $\theta=1.0^{\circ}$ with $0.001^{\circ}$ step size. Data collection time at each point was $20 \mathrm{sec}$.

The experimental reflectivity and the fluorescence yield data (Ti $K_{\alpha}, \mathrm{Fe} K_{\alpha}$, and $\mathrm{W} L_{\alpha}$ ) from the marker layers and the corresponding fitted theoretical curves are shown in Fig. 2. Although the marker layers are thin, we treated them as individual layers in the computation. The footprint correction has been incorporated in the experimental curves. All the theoretical curves in Fig. 2 for the fluorescence yield have also been corrected for the fact that the fluorescence count increases as $\theta$ increases due to finite aperture of the $\mathrm{Si}(\mathrm{Li})$ detector. The reflectivity curve does not follow the trend as calculated for the case of the Si/Au system [Fig. 1(a)]. This is because the marker layers of high electron density are also contributing to the shape of the reflectivity curve. From the fitting of the reflectivity (up to $\theta=1^{\circ}$ ) we obtain layer densities, thicknesses and surface and interface roughnesses. The parameters obtained for each Si layer are as follows: $\delta_{\mathrm{Si}}$ $=3.7 \times 10^{-6}$ and thickness $d_{\mathrm{Si}}=12.7 \pm 0.3 \mathrm{~nm}$. The $\delta$ values for $\mathrm{Ti}, \mathrm{Fe}, \mathrm{W}$, and $\mathrm{Au}$ are obtained as $6.9 \times 10^{-6}, 1.18$ $\times 10^{-5}, 2.25 \times 10^{-5}$, and $2.26 \times 10^{-5}$, respectively. The thicknesses for the Ti, Fe, W, and Au layers come out to be $1.7 \pm 0.2,1.5 \pm 0.2,1.1 \pm 0.1$, and $72.0 \pm 0.5 \mathrm{~nm}$, respectively. Surface and interface roughnesses $\left(\sigma_{j}{ }^{\prime} \mathrm{s}\right)$ are $<0.3 \mathrm{~nm}$. It is observed that the density of the layers are $4-7 \%$ smaller compared to the bulk values. Since the marker layers are of higher density than the spacer Si layers, the shape of the reflectivity curve has changed compared to an ideal Si layer of $50 \mathrm{~nm}$ thickness. We notice that the reflectivity curve [Fig. 2(a) ] has a hump at about $\theta=0.3^{\circ}$. This is because of the first order Bragg reflection from a semiperiodic multilayer formed with $\mathrm{Si}(12.7 \mathrm{~nm}) /$ marker $(\sim 1.5 \mathrm{~nm})$ bilayers. This bilayer is repeated four times on the Au layer. The Bragg peak is broad because the number of bilayers is small.

Figures 2(b), 2(c), and 2(d), show the fluorescence yield from $\mathrm{Ti}, \mathrm{Fe}$, and $\mathrm{W}$ marker layers, respectively. We fit the fluorescence yield data with the density, thickness, surface and interface roughness values extracted from the reflectivity fit. The contributions of interface roughnesses are considered as error function distributions. ${ }^{12}$ It is clear from Figs. 2(b) and 2(d) that the angular dependence of the fluorescence yield from equidistant positions around the middle of the film are quite distinct. Each of them is again distinct from the

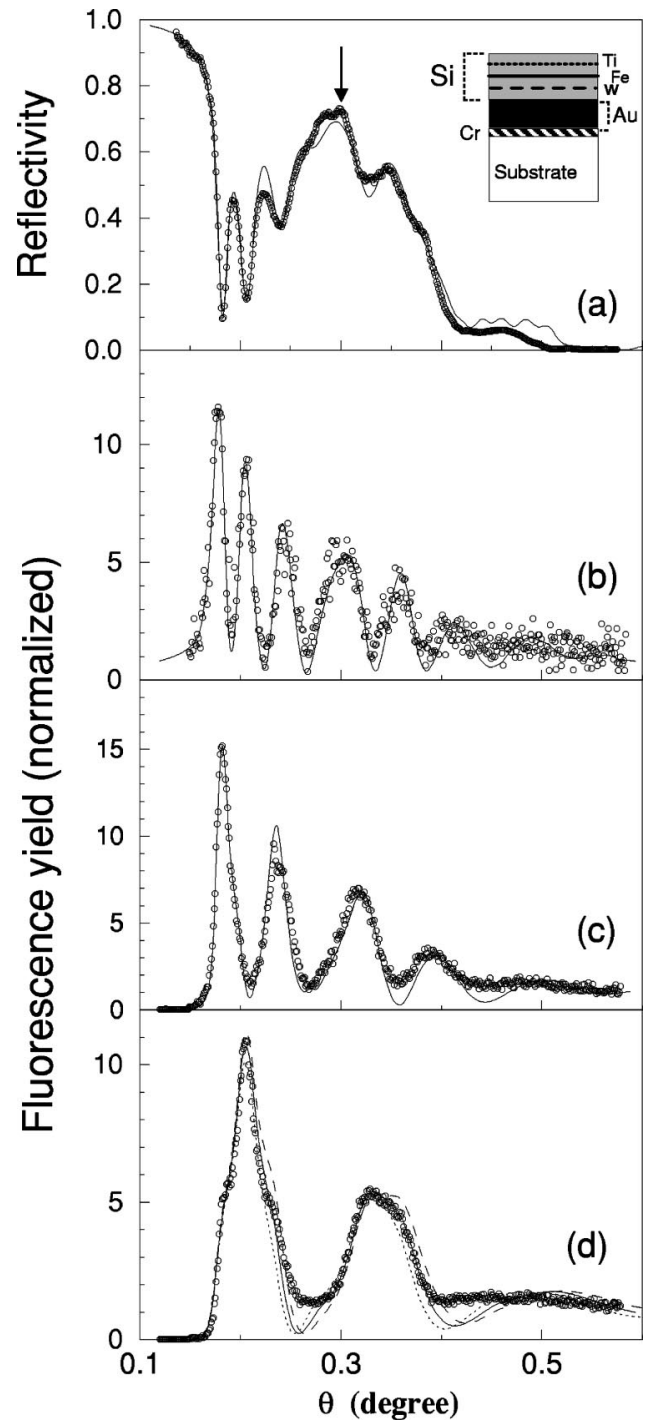

FIG. 2. Experimental data $(\bigcirc \bigcirc \bigcirc \bigcirc)$ and the fitted theoretical curves (-) for the $\mathrm{Si} / \mathrm{Ti} / \mathrm{Si} / \mathrm{Fe} / \mathrm{Si} / \mathrm{W} / \mathrm{Si} / \mathrm{Au} / \mathrm{Cr} / \mathrm{Si}$ (substrate) system, as shown in the inset. (a) Reflectivity; (b), (c), (d) are fluorescence yields from the marker layers $\mathrm{Ti}, \mathrm{Fe}$, and $\mathrm{W}$, respectively. In (d) two additional curves are given to show the depth resolution. Fluorescence yield curves for positions of $0.5 \mathrm{~nm}$ deeper $(---)$ and $0.5 \mathrm{~nm}$ shallower $(\ldots \ldots \ldots)$ from the mean best-fit position. In (a) the arrow mark shows the position of the first order Bragg peak from the semiperiodic multilayer on $\mathrm{Au}$. See text for details. Fluorescence yield data have been normalized at $\theta=1^{\circ}$. Data are presented only up to $\theta=0.6^{\circ}$ for retaining the clarity of various features.

yield from marker at the middle position [Fig. 2(c)]. This means that if a single marker layer is used within a thin film for diffusion studies, the marker layer positions for marker movement throughout the depth of the film can be unambiguously determined. In Fig. 2 we also notice $\sim 11-16$ times x-ray intensity enhancement in the first order. In Fig. 2(d), fluorescence yield curves for two positions- $0.5 \mathrm{~nm}$ deeper and $0.5 \mathrm{~nm}$ shallower than the best-fit mean position of the W layer-are shown. The fluorescence yield curves are easily distinguishable. This shows that the depth resolu- 
tion is better than $0.5 \mathrm{~nm}$. Resonance enhancement of $\mathrm{x}$ rays can be observed in materials where absorption of incident $\mathrm{x}$ rays is reasonably small. Elements or compounds involving low- $Z$ atoms fulfill this criterion. This enhancement has been shown for a polymer layer. ${ }^{7}$ Thus for diffusion studies in polymer layers using marker, REXFS would be superior to other available techniques. The superior depth resolution allows determination of diffusion coefficients as small as $10^{-21} \mathrm{~cm}^{2} / \mathrm{sec}^{13}$ In diffusion experiments marker layer movement may also broaden the marker atom distribution. The shape of the fluorescence yield curve is sensitive to this broadening and can be evaluated as in Ref. 13 .

We discuss some other areas of applications of this technique below. Novel organic/inorganic nanocomposites can be designed using simple polymers ${ }^{14}$ or using templates formed with block copolymers. These copolymers spontaneously self-assemble into regular arrays of domains whose size can be controlled from a few to several tens of nanometers. Selective decoration or loading of such block copolymer domains with metal ${ }^{15-17}$ or semiconductor ${ }^{18}$ nanoparticles can be achieved. It is extremely difficult to probe the depth profile of the nanoparticles particularly for a small volume fraction of nanoparticles. X-ray reflection standing wave fluorescence spectrometry has been applied to this problem. ${ }^{19}$ In usual x-ray standing wave experiments a maximum field intensity enhancement of four times can be achieved. However, the resonance enhancement process can achieve a much higher enhancement. In the present example as much as 16 times enhancement in the first order is seen in Fig. 2(c). For depth profile measurements the resonance enhancement technique has been used to determine depth profiles of components in a two-component polymer blend layer. ${ }^{7}$ Because of the intensity enhancement, REXFS would have a higher sensitivity for this type of applications. Together with the position resolution better than $0.5 \mathrm{~nm}$ this technique is expected to find applications in the area of nanocomposites.

Our experiment, utilizes the simultaneous detection of elastic scattering (reflection) and one of the inelastic scattering processes, fluorescence. Detection of other inelastic scattering processes can provide other valuable information. For example, in some cases phonon spectra from confined nanoparticles can be obtained via inelastic nuclear resonant scattering from nanoparticles containing Mössbauer isotopes. Such experiments on thin films containing ${ }^{57} \mathrm{Fe}$ Mössbauer isotopes have been performed by Röhlsberger et al. ${ }^{20}$ However, they could achieve an intensity enhancement of about 4 times. This is because the marker atoms were distributed over the whole thickness. A comparison of the response from distributed marker layer and that from a marker layer confined in the middle of the film is given in Ref. 7. On a thin Fe layer, confined at the middle of a polymer or silicon layer, as shown here, the field enhancement is much larger. In the present case it is 19 times enhanced [Fig. 1(b)]. An optimized condition would give even higher enhancements. The enhanced sensitivity due to this $\mathrm{x}$-ray intensity enhancement would allow measurements on an amount of materials much smaller than a monolayer. This would mean that in an iron film, the surface vibrational density of states can be distinguished from that in the bulk of the layer by selectively putting a ${ }^{57} \mathrm{Fe}$ monolayer at the surface or in the middle of the Fe layer.

In summary, we have shown that in resonance enhanced $\mathrm{x}$-ray fluorescence spectometry experiments, position of a marker layer within a thin film can be determined unambiguously. Thus diffusion studies using marker layers can be performed in thin films of polymers and other materials involving low- $Z$ atoms. The $x$-ray intensity enhancement would provide higher sensitivity. Combined with its superior depth resolution, we believe that this technique will find applications in the areas of polymer and nanostructure research.

\section{ACKNOWLEDGMENTS}

We thank S. K. Poddar and S. M. Chaudhury for the sample preparation, G. Kuri for helping with the experiment, and G. Materlik for providing his experimental facilities. S.K.G. and B.N.D. acknowledge the financial support of the Department of Science and Technology, Govt. of India, under the VISTA program (VISTA-1/2000).

*Email: bhupen@iopb.res.in; FAX: +91-674-300142.

†Present address: Department of Physics, University of Illinois, Urbana, IL 61801, USA.

${ }^{1}$ A. H. Compton and S. K. Allison, X-rays in Theory and Experiment (Van Nostrand, New York, 1935).

${ }^{2}$ H. Kiessig, Ann. Phys. (Leipzig) 10, 769 (1931).

${ }^{3}$ P. Eisenberger and W. C. Marra, Phys. Rev. Lett. 46, 1081 (1981).

${ }^{4}$ R. S. Becker, J. A. Golovchenko, and J. R. Patel, Phys. Rev. Lett. 50, 153 (1983).

${ }^{5}$ M. J. Bedzyk, G. M. Bommarito, and J. S. Schildkraut, Phys. Rev. Lett. 62, 1376 (1989).

${ }^{6}$ J. Wang, M. J. Bedzyk, and M. Caffrey, Science 258, 775 (1992).

${ }^{7}$ B. N. Dev, Amal K. Das, S. Dev, D. W. Schubert, M. Stamm, and G. Materlik, Phys. Rev. B 61, 8462 (2000).

${ }^{8}$ D. K. G. De Boer, Phys. Rev. B 44, 498 (1991).

${ }^{9}$ P. F. Green, C. J. Palmstrom, J. W. Mayer, and E. J. Kramer, Macromolecules 18, 501 (1985).

${ }^{10}$ G. Reiter, S. Huttenbach, M. Foster, and M. Stamm, Macromolecules 24, 1179 (1991).

${ }^{11}$ L. G. Parratt, Phys. Rev. 95, 359 (1954).

${ }^{12}$ S. K. Ghose and B. N. Dev, Phys. Rev. B 63, 245409 (2001).

${ }^{13}$ S. Dev and B. N. Dev, Indian J. Phys., A 70, 189 (1996); 70, 543 (1996).

${ }^{14}$ Amal K. Das, J. Kamila, B. N. Dev, B. Sundaravel, and G. Kuri, Appl. Phys. Lett. 77, 951 (2000).

${ }^{15}$ T. L. Morkved, P. Witzius, H. M. Jaeger, D. G. Grier, and T. A. Witten, Appl. Phys. Lett. 64, 422 (1994).

${ }^{16}$ R. W. Zehner et al., Langmuir 14, 241 (1998).

${ }^{17}$ D. H. Cole, K. R. Shull, L. E. Rehn, and P. Baldo, Phys. Rev. Lett. 78, 5006 (1997).

${ }^{18}$ D. E Fogg et al., Macromolecules 30, 417 (1997).

${ }^{19}$ B. Lin et al., J. Appl. Phys. 85, 3180 (1999).

${ }^{20}$ R. Röhlsberger et al., Physica B 263-264, 581 (1999); J. Appl. Phys. 86, 584 (1999). 